\title{
Abundancia y distribución epipelágica de paralarvas (Cephalopoda: Mollusca) en el Pacífico Colombiano: periodos Iluviosos 2001-2004
}

\section{Epipelagic abundance and distribution of paralarvae (Cephalopoda: Mollusca) in the Colombian Pacific: rainy periods 2001-2004}

\author{
Eileen Paola Vargas ${ }^{1}$; Raúl Hernando López ${ }^{2}$
}

'Bióloga, Esp. Instituto Geográfico Agustín Codazzi. Bogotá, Colombia, e-mail: eileenpao@hotmail.com; (D) https://orcid.org/0000-0002-3906-1789

²Biólogo Marino, M.Sc., Ph.D. Universidad Militar Nueva Granada, Facultad de Ciencias Básicas y Aplicadas, Programa de Biología Aplicada. Cajicá Cundinamarca, Colombia, e-mail: raul.lopez@unimilitar.edu.co; (D) https://orcid.org/0000-0002-7996-1319

Cómo citar: Vargas, E.P.; López, R.H. 2020. Abundancia y distribución epipelágica de paralarvas (Cephalopoda: Mollusca) en el Pacífico Colombiano: periodos Iluviosos 2001-2004. Rev. U.D.C.A Act. \& Div. Cient. 23(1):e1528. http://doi.org/10.31910/rudca.v23. n1.2020.1528

Artículo de acceso abierto publicado por Revista U.D.C.A Actualidad \& Divulgación Científica, bajo una licencia Creative Commons CC BY-NC 4.0

Publicación oficial de la Universidad de Ciencias Aplicadas y Ambientales U.D.C.A, Institución de Educación Superior Acreditada de Alta Calidad por el Ministerio de Educación Nacional.

Recibido: Junio 20 de 2019

Aceptado Abril 22 de 2020

Editado por: Ingeborg Zenner de Polanía

\section{RESUMEN}

Pese a su gran importancia ecológica y económica, la información sobre los cefalópodos es exigua, en especial, sobre sus paralarvas, particularmente, en el Océano Pacífico Colombiano (OPC), por lo tanto, se evaluó su abundancia y distribución en los periodos 23/ jun-12/jul/01, 27/ago-15/sep/01, 03-22/sep/02, 01-21/sep/03 y 18/sep-08/oct/04. Los organismos, se pescaron en la superficie con una red cónica $(\varnothing=50 \mathrm{~cm}$, longitud $1,85 \mathrm{~m}$, poro $363 \mu \mathrm{m})$. Los datos de temperatura y de salinidad se obtuvieron con una sonda CTD. Se identificaron Ommastrephidae (66,3\%), AncistrocheiridaeAncistrocheirus lesueuri (14,2\%) (primer registro específico paralarval para el OPC), Cranchiidae (1,5\%), Enoploteuthidae, Gonatidae, Octopodidae, Onycoteuthidae y Pyroteuthidae $(\leq 0,5 \%)$. Las paralarvas recien eclosionadas $(\leq 2,0 \mathrm{~mm})$ abarcaron todo el OPC, indicando su relevancia como área de desove, especialmente, en aguas neríticas. El menor tamaño al eclosionar, se podría deber a las mayores temperaturas en el OPC que en otras latitudes del Pacífico Americano. La distribución meso-escalar pudo estar mediada, en principio, por el giro geostrófico, la Corriente Sur Ecuatorial y la Corriente de Colombia. El Niño-moderado, quizás influyó en la aparición exclusiva de Ommastrephidae durante el periodo 03-22/sep/02. El predominio nocturno (83,2 vs. 16,8\% día), se atribuyó a las migraciones verticales de las paralarvas. La variabilidad de estos hallazgos pudo estar relacionada con los cambios ambientales inter-anuales, enmarcados en la gran complejidad ambiental del OPC y otros factores no sopesados, como la disponibilidad de alimento y la depredación.

Palabras clave: Pacífico colombiano; paralarvas; taxonomía; talla; El Niño. 
Vargas, E.P.; López, R.H.: paralarvas Pacífico Colombiano

\section{ABSTRACT}

Despite their great ecological and economic importance, information on cephalopods is scarce, especially on their paralarvae, particularly in the Colombian Pacific Ocean (OPC). Therefore, their abundance and distribution in the periods 23.Jun-12.Jul.01, 27.Ago-15. Sep.01, 03-22.Sep.02, 01-21.Sep.03 and 18 .Sep-08.Oct.04 were evaluated. Organims were caught at the surface with a conical net $(\varnothing=50 \mathrm{~cm}$, length $1.85 \mathrm{~m}$, pore $363 \mu \mathrm{m})$. The temperature and salinity data were obtained with a CTD probe. Ommastrephidae (66.3\%), Ancistrocheiridae-Ancistrocheirus lesueuri (14.2\%) (first specific paralarval record for the OPC), Cranchiidae (1.5\%), Enoploteuthidae, Gonatidae, Octopodidae, Onycoteuthidae, and Pyroteuthidae $(\leq 0.5 \%)$ were identified. Newly hatched paralarvae $(\leq 2.0 \mathrm{~mm})$ covered the whole OPC, indicating its relevance as a spawning area, especially in neritic waters. The smaller size at hatching could be due to the higher temperatures in the OPC than in other latitudes of the American Pacific. The meso-scalar distribution could be mediated especially by the geostrophic eddy, the Equatorial South Current and the Colombian Current. El Niño-moderate perhaps influenced the exclusive appearance of Ommastrephidae during the period 03-22.Sep.02. The night predominance (83.2 vs. $16.8 \%$ day) was attributed to the paralarval vertical migrations. The variability of these findings could be related to the interannual environmental changes framed in the great environmental complexity of the OPC and other unweighted factors, such as food availability and predation.

Keywords: Colombian Pacific; paralarvae; taxomomy; size; El Niño.

\section{INTRODUCCIÓN}

La Clase Cephalopoda Cuvier, 1797 tiene ca. 800 especies de calamares, sepias, nautilos y pulpos de distribución cosmopolita en ambientes nectónicos, demersales o bentónicos (Jereb \& Roper, 2005). Tienen un amplio espectro alimentario y son presa de peces, aves y mamíferos marinos. Se caracterizan por su rápido crecimiento con fases post-eclosión planctónicas y algunas bentónicas, denominadas paralarvas, semejantes a los adultos (Sweeney et al. 1992; Rodhouse et al. 2014). Pese a su gran plasticidad, por sus ciclos de vida cortos, los cefalópodos son especialmente sensibles a las condiciones ambientales, por lo cual, se han considerado indicadores de cambios ambientales locales. La mayoría hacen parte de la pesca artesanal e incidental de camarón o carnada de peces y pocas especies de calamares y de pulpos tienen valor comercial (Rodhouse et al. 2014).

En el Pacífico Americano, la información concerniente a las paralarvas es inexistente o rudimentaria (con varias tesis no publicadas) y se ha enfocado, especialmente, en la influencia oceanográfica sobre las familias, dada su difícil identificación específica, e.g., en el Pacífico Oriental Tropical (POT) (Vecchione, 1999), en particular, en aguas de México (Alejo-Plata et al. 2012; Alejo et al. 2013; Hendrickx et al. 2015; Aceves et al. 2017; García et al. 2018; Ruvalcaba et al. 2018) y Chile (Vega et al. 1999; Vega et al. 2000; Carrasco et al. 2012; Ibañez et al. 2012; Pardo et al. 2016) y pocos en Costa Rica (Vecchione,
1999), Perú (Cardoso et al. 2005; Ayón, 2018) y Colombia (Rosales \& López, 2007).

Esta investigación, se orientó a identificar las familias de paralarvas y a evaluar su abundancia y distribución en el OPC durante cinco cruceros oceanográficos del Estudio Regional del Fenómeno El Niño (ERFEN)-Colombia. Los resultados sirven para fortalecer las series de tiempo y comprender los cambios históricos en la comunidad paralarval, para posteriores comparaciones, a nivel local meso y macroescalar, e.g., la influencia de El Niño y la Oscilación Decadal del Pacífico, así como para el análisis del posible desarrollo futuro de las pesquerías de cefalópodos en el OPC, en vista de que la infomación sobre sobre la captura, en especial, de calamares adultos, es incipiente (De la Hoz et al. 2017).

\section{MATERIALES Y MÉTODOS}

Área de estudio. El OPC $\left(01^{\circ} 30^{\prime}-06^{\circ} 30^{\prime}\right.$ latitud norte, $77^{\circ} 45^{\prime}-84^{\circ} 00^{\prime}$ longitud oeste, $c a .349 .000 \mathrm{Km}^{2}$ ) (Figura 1a-b), está influenciado por los desplazamientos de la Zona de Convergencia Intertropical, los vientos Alisios del norte y sur y los eventos El Niño y La Niña. Los flujos superficiales mesoéscalares son la Corriente Sur Ecuatorial, más intensa en el segundo semestre del año o periodo cálido lluvioso, en particular, en septiembre; las Corrientes de Panamá, Colombia (paralela a la costa con remolinos ciclo- y anticiclónicos), y Chocó, y surgencias costeras y oceánicas todo el año, particularmente entre agosto y septiembre. Son notorios el amplio régimen mareal y la elevada descarga dulcecuícola costero-nerítica (CCCP, 2002; Devis, 2003; Corredor et al. 2011).

Obtención de las paralarvas y los datos abióticos. Las muestras, se obtuvieron en cinco cruceros oceanográficos: 23/jun-12/jul/01, 27/ago-15/sep/01, 03-22/sep/02, 01-21/sep/03 y 18/sep-08/ oct/04. Se mantuvo la numeración original de las 113 estaciones del Programa ERFEN-Colombia (Figura 1). Su número/campaña dependió de factores logísticos y las condiciones climáticas, asî que no fue posible contar con un grupo total homogéneo de estaciones en número, ubicación y hora de las faenas. Los arrastres de zooplancton, se efectuaron en el primer metro superficial, usando una red cónica $(\varnothing=50 \mathrm{~cm}$, longitud $1,85 \mathrm{~m}$ y poro $363 \mu \mathrm{m})$, provista de un flujómetro General Oceanics, para determinar el volumen de agua filtrada. Las muestras, se fijaron en solución de agua de mar con formaldehído al 10\% y se conservaron en formaldehído al 4\%; en ambos casos, neutralizado con tetraborato de sodio. Los datos de temperatura $\left({ }^{\circ} \mathrm{C}\right)$ y de salinidad (Unidades Prácticas de Salinidad= UPS) fueron medidos con una sonda CTD (Conductividad, Temperatura, Depth= profundidad), Seabirds Electronics.

Identificación. De cada muestra de zooplancton, se extrajeron todas las paralarvas, identificándolas con base en su habitus y longitud del manto (LM mm) (micrómetro ocular de 0,01 mm-microscopio), presencia de aletas y probóscide, número de brazos y tentáculos, tipo de ojos y número y posición de los cromatóforos (Sweeney et al. 1992; Alejo et al. 2013). 


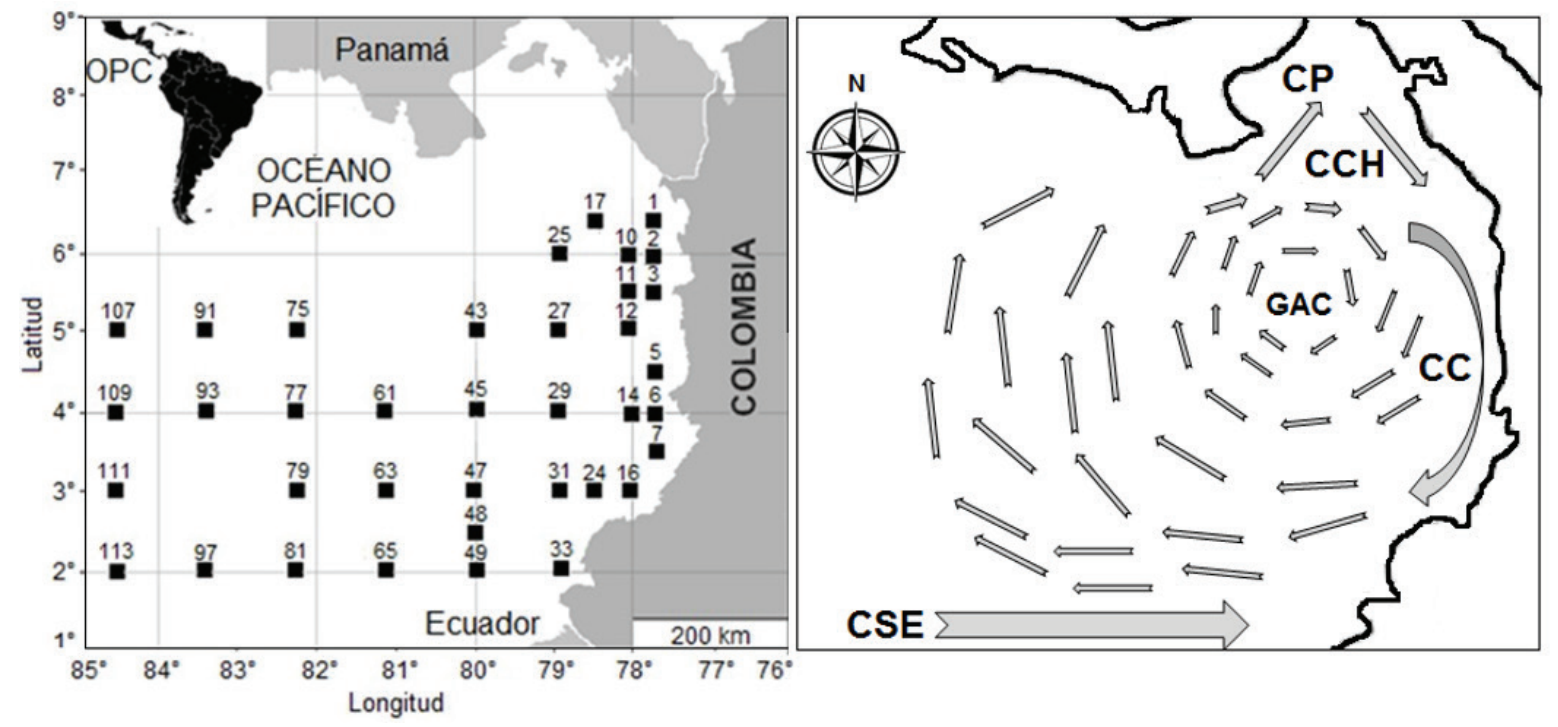

Figura 1. a. Estaciones ERFEN-Colombia para el estudio de las paralarvas de cefalópodos; b. Esquema simplificado de las corrientes superficiales en el OPC (basado en Corredor et al. 2011). CSE= Corriente Sur Ecuatorial, CC= Corriente de Colombia, GAC= Giro Anticiclónico, $\mathrm{CP}=$ Corriente de Panamá, $\mathrm{CCH}=$ Corriente del Chocó.

Análisis de la información. La abundancia paralarval (Pl/estación), se estimó según la fórmula: $\mathrm{Pl}=1000 \mathrm{~m}^{3} \cdot \mathrm{Pl} / \pi \cdot \mathrm{r}^{2} \cdot \mathrm{d}$, donde: $\mathrm{Pl}=$ individuos contabilizadas, $\mathrm{r}=$ radio de la red, $\mathrm{d}=$ distancia del arrastre, $\pi \cdot r^{2} \cdot d=$ volumen de agua filtrada (Smith \& Richardson, 1979). Los rangos de baja, media y alta abundancia, se definieron mediante cuartiles, previa transformación con log $(x+1)$. Para evaluar las asociaciones entre la abundancia paralarval, la temperatura, la salinidad y la abundancia día (06:00-18:00h) vs. noche (18:01-05:59h), se realizaron análisis de agrupamiento y factores, en este caso, por el método de componentes principales y rotación ortogonal varimax de los factores, a fin de homogenizar su distribución y la varianza explicada por factor. Ante la ausencia de normalidad (Shapiro-Wilk) y homocedasticidad (Bartlett), se aplicó la prueba de Mann-Whitney (U), con el fin de cotejar diferencias y semejanzas de las variables abióticas y la abundancia entre las subáreas nerítica, intermedia y oceánica, así como la prueba de Kruskal-Wallis $(\mathrm{p}<0,05)$, para la comparación inter-periodos (en ambos casos, nivel de significancia $<0,05)$. Se usó el programa SPSS Statistics 20®.

\section{RESULTADOS Y DISCUSIÓN}

Estructura termo-halina en la superficie del OPC. En general, la salinidad exhibió su amplio rango (24,8-35,0UPS) y distribución usual, con valores más bajos hacia la zona nerítica, como consecuencia de la alta pluviosidad propia del OPC y el gran número de ríos (CCCP, 2002), en contraste con el rango térmico más estrecho $\left(24,4-29,7^{\circ} \mathrm{C}\right)$ y temperaturas más altas en la misma zona (Figura 2a), definiendo un gradiente termohalino inverso costa-océano. La temperatura y profundidad de la termoclina evidenciaron El Niño moderado 2002-2003, que influenció especialmente el extremo suroccidental del OPC (Devis, 2003). Este esquema, se vió reflejado estadísticamente, pues solo se detectaron diferencias significativas de la salinidad entre las sub-áreas nerítica y oceánica (U M-W: 2763; $\mathrm{p}=0,00038)$, así como de esta variable y la temperatura (K-W: $\mathrm{H}=$ 12,76; $\mathrm{p}<0,1252$ y $\mathrm{H}=45,49 ; \mathrm{p}<0,00001$, respectivamente), entre los cinco periodos evaluados.

Composición. En todos los periodos predominó el orden Teuthida Naef, 1916 (calamares) (Roper \& Jereb, 2010), en especial, la familia Ommastrephidae Steenstrup, 1857 ( $n=124 ; 66,3 \%$ ), siendo única en dos de ellos. Fueron raras Ancistrocheiridae Pfeffer, 1912 ( $\mathrm{n}=1$; 14,2\%), Cranchiidae Prosch, 1847 ( $n=3 ; 1,5 \%$ ), Enoploteuthidae Pfeffer, $1900(n=1)$ y Pyroteuthidae Pfeffer, 1912 ( $n=1 ; 0,5 \%$ cada una); Gonatidae Hoyle, 1886 y Onycoteuthidae Gray, 1847 ( $n=1$; 0,2\% cada una). Del orden orden Octopoda Leach, 1818 (pulpos) solo apareció Octopodidae D’Orbigny, 1839 ( $\mathrm{n}=1 ; 0,2 \%$ ); el 16,4\% fue nn (Figura 2a). De Ancistrocheiridae, se identificó Ancistrocheirus lesueuri (Orbigny, 1842) (18/sep-08/oct/04, una estación), especie amenzada (Allcock \& Barratt, 2014), de la cual, solo se sabe que, posiblemente, desova en el fondo, cerca del talud continental o en océano abierto, a lo largo del año (Arkhipkin, 1997). Se sospecha la existencia de otra especie en el Pacífico Americano, sin diferenciarse aún (De Silva et al. 2015).

Las paralarvas de Cranchiidae (27/ago-15/sep/01, una estación; $18 /$ sep-08/oct/04, cuatro estaciones) son comunes en aguas superficiales, donde muchas habitan hasta ca. 50-100mm LM (Voss et al. 1992). Pyroteuthidae (23/jun-12/jul/01, una estación) y Enoploteuthidae (27/ago-15/sep/01, dos estaciones), comprenden pocas especies (Ruvalcaba et al. 2018), disminuyendo la probabilidad de capturar sus paralarvas. Tienen estrategias reproductivas y de desove similares (Young et al. 1992), aunque su hábitat difiere en profundidad (Ruvalcaba et al. 2018), lo que sustenta su aparición separada en los dos periodos de 2001, mientras que su coexistencia 


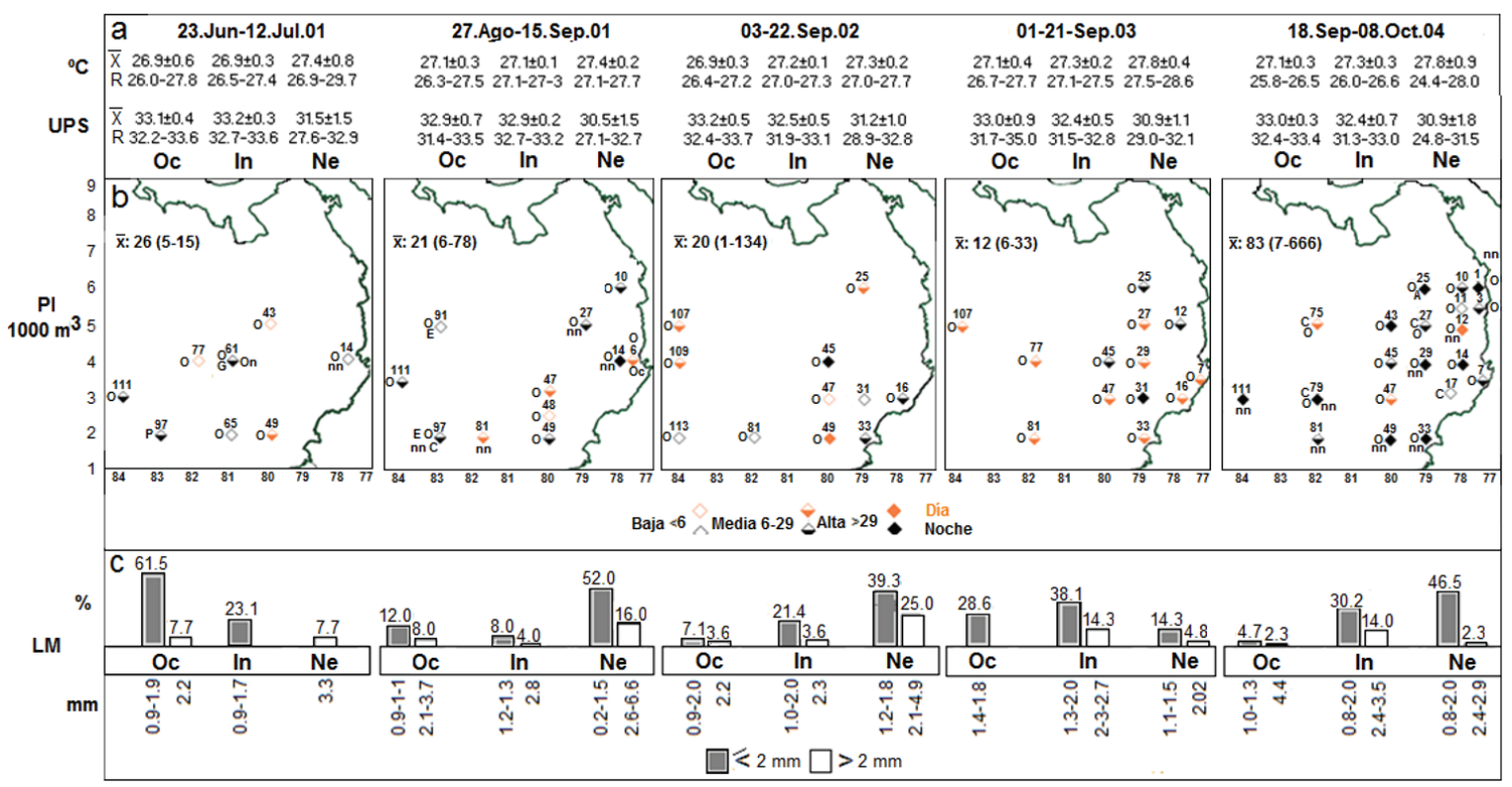

Figura 2. a-b. Temperatura $\left({ }^{\circ} \mathrm{C}\right)$, salinidad (UPS) y abundancia paralarval (Pl/1000 $\mathrm{m}^{3}$ ) por periodo evaluado en el OPC. c. Abundancia de paralarvas (\%) según la LM (mm). Sub-áreas: $\mathrm{Ne}=$ nerítica, $\mathrm{In}=$ Intermedia, Oc= Oceánica. $\mathrm{R}=$ rango. $\mathrm{O}=\mathrm{Ommastrephidae,} \mathrm{A=}$ Ancistrocheiridae, $\mathrm{C}=$ Cranchiidae, $\mathrm{E}=$ Enoplotheutidae, $\mathrm{G}=$ Gonatidae, Oc= Octopodidae, On= Onychotheutidae, $\mathrm{P}=\mathrm{Pyrotheutidae}$. No se incluyen las estaciones con ausencia de paralarvas.

en sep/05 (Rosales \& López, 2007), se atribuye al muestreo oblícuo. La exigua aparición de Octopodidae (27/ago-15/sep/01, una estación), se explica por el rápido asentamiento en el mismo ambiente bentónico-nerítico-costero de los adultos, con menor potencial de dispersión que los cefalópodos pelágicos, cuya vida planctónica varía de días a meses (Vega et al. 2000; Carrasco et al. 2012; Rodhouse et al. 2014; Villanueva et al. 2016; De Silva et al. 2015; García et al. 2018; Ruvalcaba et al. 2018). Las paralarvas de Gonatidae suelen habitar los primeros 200m (Bower \& Takagi, 2004; Jorgensen, 2007) y las de Onychoteuthidae hasta los $500 \mathrm{~m}$ (Roper \& Jereb, 2010), explicando su captura en sendas estaciones (27/ago-15/sep/01).

En sep/05, Rosales \& López (2007) hallaron Ommastrephidae (61,0\%), Cranchiidae (13,0\%), Enoploteuthidae, Pyroteuthidae, Bolitaenidae, Oegopsina, Onychoteuthidae y Octopodidae $(\leq 6,0 \%)$. Aunque solo consideraron cuatro estaciones, las semejanzas/ disimilitudes con el presente estudio, se podrían deber a las condiciones ambientales diferentes y los muestreos oblicuos $(\overline{\mathrm{Z}}=$ $160 \mathrm{~m})$, en 2005 .

Pese a la dificultad para identificar las paralarvas (Rodhouse et al. 2014; Ruvalcaba et al. 2018), la lista de las ocho familias registradas en el OPC es considerable, comparada con otras regiones del Pacífico Americano, que totalizan 19 familias, con variaciones en la abundancia y la LM (Tabla 1), así como en la ocurrencia, dependiendo de la época del año, dinámica oceanográfica, número de estaciones y tipo de muestreo. Una forma de contribuir con estos análisis es conociendo la distribución de los calamares adultos por taxa (Boyle \& Rodhouse, 2005; Rodhouse et al. 2014), lo que, eventualmente, pudiera corresponder a algunos de los grupos de paralarvas capturados, pero en Colombia, los registros sobre su pesca, en especial, en el Pacífico colombiano, son esporádicos y puntuales y solo mencionan las especies Loliolopsis diomedeae y Dosidicus gigas, sin especificar meses, ni lugares de captura (De la Hoz et al. 2017).

Abundancia y distribución. Se realizaron 133 arrastres, 64 con paralarvas, cuyo bajo número $\left(\sum=213\right)$ concuerda con lo observado en otras regiones (Granados et al. 2010; Alejo et al. 2013; De Silva et al. 2015; Hendrickx et al. 2015; Aceves et al. 2017; García et al. 2018; Ruvalcaba et al. 2018), relacionado con su habilidad para esquivar las redes (Alejo et al. 2013).

En orden cronológico, los promedios fueron similares en los tres primeros periodos $\left(26,21\right.$ y $20 \mathrm{Pl} / 1000 \mathrm{~m}^{3}$, respectivamente), mientras en el tercero fue el más bajo $\left(12 \mathrm{Pl} / 1000 \mathrm{~m}^{3}\right)$ y, en el último, el más elevado (83 Pl/1000m ${ }^{3}$ ) (Figura 2b); 56,98\% de las paralarvas, se registró en aguas intermedias, quizás por el transporte y la concentración en el giro anticiclónico imperante allí (CCCP, 2002; Corredor et al. 2011) o en combinación con condiciones ambientales de transición costa-océano; 26,27\% correspondió a aguas neríticas, donde son comunes las surgencias, remolinos ciclónicos y anticiclónicos a lo largo de la Corriente de Colombia (CCCP, 2002; Corredor et al. 2011) y 10,29\% en aguas oceánicas, influenciadas por surgencias y el ramal occidental del giro anticiclónico, que pudo transportar paralarvas a esa subárea, donde se ubica la isla Malpelo, hábitat de pulpos (Domínguez et al. 2013) 


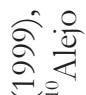

:

惫

$\stackrel{3}{2}$

莺

* $\frac{1}{*} \cdot \frac{1}{d}$

वृं

疍

乙े

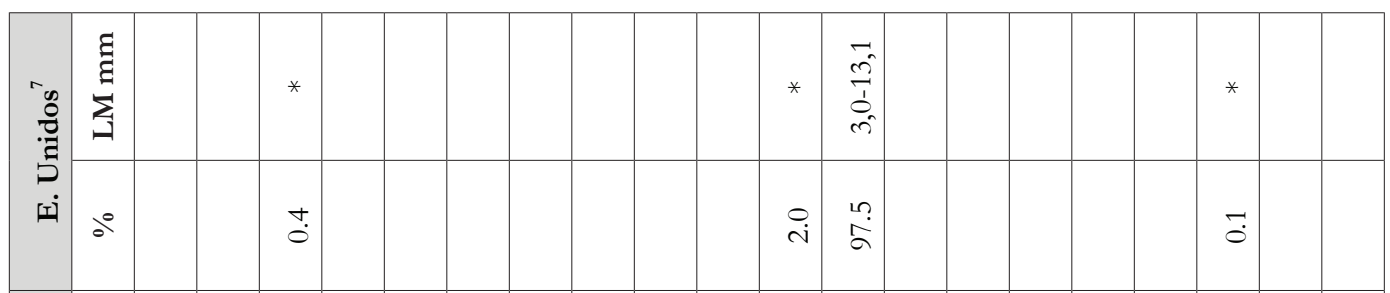

$11 \pm$

:

등

全过

में

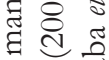

च चี तु

च

50

용

푱

न वृ

व 2

तै ธ

式

दे है

ช०

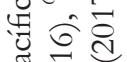

$\stackrel{2}{2}$

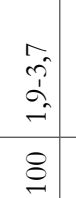

(3.

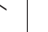

वे वे

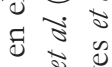

苗 :

可?

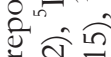

홍리

告

क्षे के

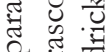

记

过

पิ

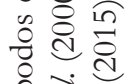

.ำ वें

跑芯

ช 5

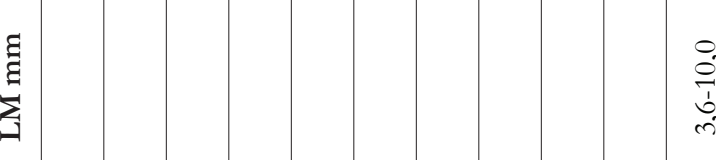

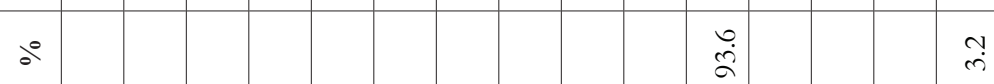

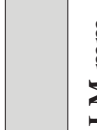

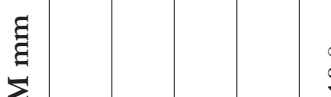

ปี

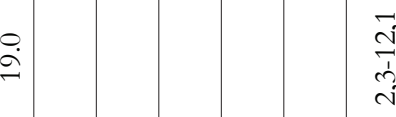

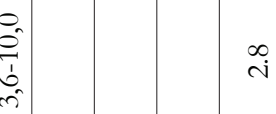

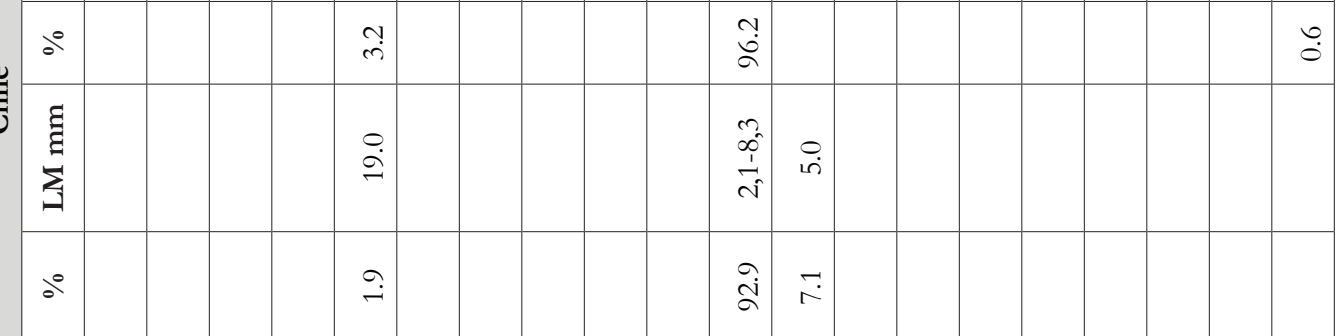

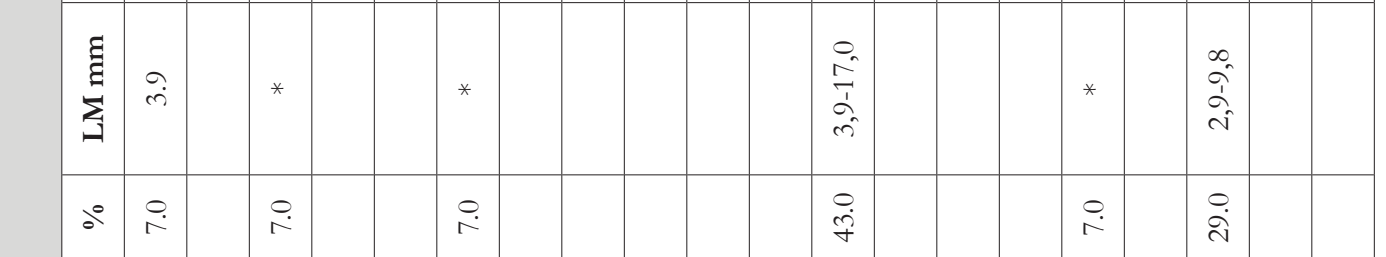

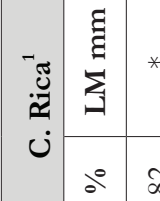

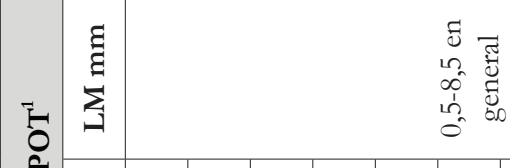

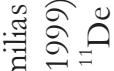

द्व $=$

एक

- ¿ ह

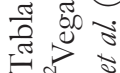

\begin{tabular}{|c|c|c|c|c|c|c|c|c|c|c|c|c|c|c|c|c|c|c|c|c|}
\hline 胥 & & & & & & & & & & & & & & & & & & & & \\
\hline$\partial^{\circ}$ & $\stackrel{+}{\circ}$ & $\stackrel{+}{0}$ & $\stackrel{5}{\circ}$ & $\stackrel{\circ}{\circ}$ & $\stackrel{t}{0}$ & $\stackrel{\sigma}{0}$ & $\stackrel{\Xi}{\circ}$ & $\stackrel{\overrightarrow{0}}{\circ}$ & $\tilde{\delta}_{0}$ & $\overline{8}_{0}$ & $\overline{8}$ & & & & & & & & & \\
\hline 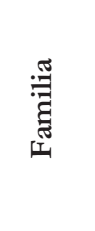 & 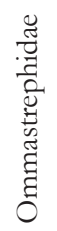 & 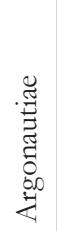 & 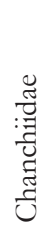 & 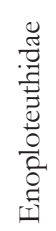 & 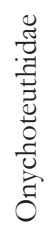 & 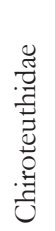 & 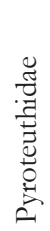 & 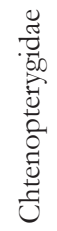 & 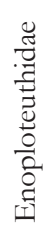 & 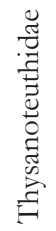 & 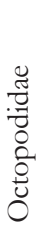 & 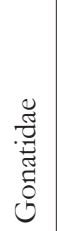 & 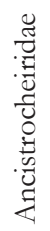 & 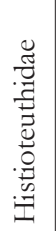 & 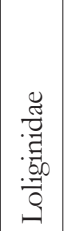 & 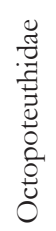 & 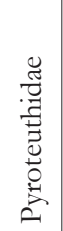 & 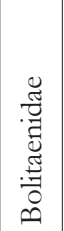 & 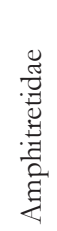 & 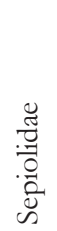 \\
\hline
\end{tabular}




\begin{tabular}{|c|c|c|c|c|c|c|c|c|c|c|c|c|c|c|c|c|c|c|c|c|}
\hline \multirow{16}{*}{ 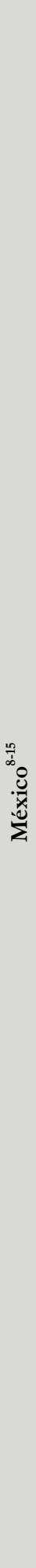 } & 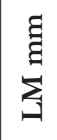 & \multicolumn{17}{|c|}{ 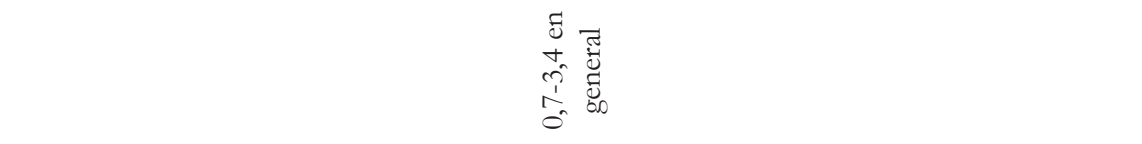 } & & \\
\hline & $a^{\circ}$ & 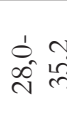 & & & & & & & & 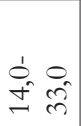 & & $\begin{array}{l}0 \\
\hat{n} \\
0 \\
0 \\
0 \\
0\end{array}$ & & & & & & 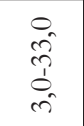 & & \\
\hline & 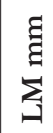 & 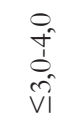 & $*$ & $*$ & & $*$ & $*$ & & & * & & & * & * & & & $*$ & * & & $*$ \\
\hline & $a^{\circ}$ & ஜ & $\dot{\vec{r}}$ & $\stackrel{+}{0}$ & & $\stackrel{乛}{\rightarrow}$ & $\stackrel{1}{0}$ & & & $\stackrel{\vec{g}}{\sim}$ & & & 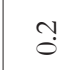 & 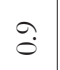 & & & $\stackrel{\infty}{i}$ & $\ddot{n}$ & & $\stackrel{\infty}{\rightarrow}$ \\
\hline & 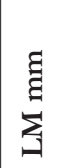 & $\begin{array}{l}\hat{0} \\
\hat{\lambda} \\
\hat{a} \\
\hat{0} \\
\hat{v i}\end{array}$ & & $\stackrel{0}{\hat{\lambda}}$ & & $\begin{array}{l}0 \\
\hat{\lambda} \\
\hat{0} \\
\hat{0} \\
\hat{v i}\end{array}$ & $\begin{array}{l}0 \\
\hat{N} \\
\hat{0} \\
\hat{\tilde{v}} \\
\mathrm{vi}\end{array}$ & & & $\begin{array}{l}\hat{0} \\
\hat{i} \\
\hat{a} \\
\hat{i} \\
v i\end{array}$ & & & & & & * & * & * & & \\
\hline & $a^{\circ}$ & $\stackrel{\overbrace{}}{\stackrel{+}{*}}$ & & $\stackrel{+}{\infty}$ & & $\underset{\infty}{\rightarrow}$ & $\stackrel{\circ}{\dot{r}}$ & & & $\stackrel{+}{\dot{c}}$ & & & & & & * & $*$ & * & & \\
\hline & 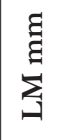 & & & & & & & & & 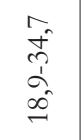 & & & & & & & & 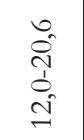 & & \\
\hline & $0^{\circ}$ & & & 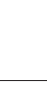 & & 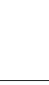 & & & & $\begin{array}{l}\infty \\
\text { a } \\
\end{array}$ & & & & & & & & $\stackrel{N}{\curvearrowright}$ & & \\
\hline & $\sum_{\text {J }}^{\xi}$ & $\begin{array}{l}0 \\
\dot{d} \\
0 \\
\hat{v} \\
v i\end{array}$ & $*$ & & & * & & & & $\begin{array}{l}\infty \\
\hat{n} \\
\hat{o} \\
\stackrel{n}{n}\end{array}$ & & * & 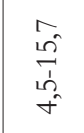 & $\begin{array}{l}0 \\
\stackrel{0}{0} \\
0^{\prime} \\
i\end{array}$ & & & & * & $*$ & \\
\hline & $a^{\circ}$ & $\begin{array}{ll}1 & 0 \\
0 & 8 \\
\forall & 18\end{array}$ & $\stackrel{\circ}{\stackrel{\infty}{n}}$ & & & $*$ & & & & $\begin{array}{l}\hat{i} \\
\hat{\vec{v}}\end{array}$ & & $\stackrel{\circ}{\stackrel{0}{n}}$ & $\begin{array}{cc}1 & 0 \\
m & i\end{array}$ & $*$ & & & & $\begin{array}{ll}\dot{1} & 0 \\
\dot{d} & + \\
\sim & +\end{array}$ & $*$ & \\
\hline & 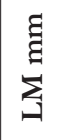 & 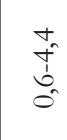 & $\begin{array}{l}\vec{r} \\
\stackrel{1}{r} \\
\vec{r} \\
\rightarrow\end{array}$ & $\begin{array}{l}\infty \\
\stackrel{0}{a} \\
\hat{n} \\
\vec{v}\end{array}$ & & $\begin{array}{l}m \\
n^{n} \\
n \\
n \\
0\end{array}$ & & & & $\begin{array}{l}0 \\
\hat{b} \\
b_{0}^{\prime} \\
0^{2}\end{array}$ & & $\begin{array}{l}m \\
\stackrel{n}{d} \\
\stackrel{j}{o}\end{array}$ & & & & & & & & \\
\hline & $\partial^{\circ}$ & $\stackrel{n}{=}$ & $\stackrel{?}{\sigma}$ & F & & $\hat{\sigma}$ & & & & மे & & $\begin{array}{l}n \\
\infty \\
\infty \\
f\end{array}$ & & & & & & & & \\
\hline & 吾 & & & & & & & & & & & $\begin{array}{l}\hat{\hat{N}} \\
\hat{1} \\
\hat{0}\end{array}$ & & & & & & & & \\
\hline & $a^{\circ}$ & & & & & & & & & & & 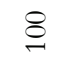 & & & & & & & & \\
\hline & 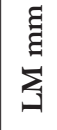 & $\hat{\tilde{v}}$ & & $*$ & $*$ & $*$ & & & & & & & * & & & & & & & \\
\hline & $a^{\circ}$ & $\stackrel{\circ}{\mathrm{i}}$ & & $\stackrel{\circ}{i}$ & $\begin{array}{l}\infty \\
\infty \\
0\end{array}$ & $\stackrel{\circ}{\bullet}$ & & & & & & & $\stackrel{\circ}{\circ}$ & $\begin{array}{l}\infty \\
\stackrel{0}{0} \\
\stackrel{0}{0}\end{array}$ & $\stackrel{\infty}{\infty}$ & $\stackrel{\infty}{\infty}$ & $\stackrel{\infty}{\infty}$ & $\stackrel{\infty}{\infty}$ & & \\
\hline & हี & 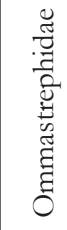 & 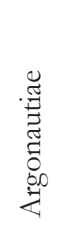 & 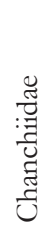 & 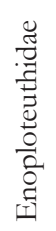 & 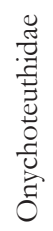 & 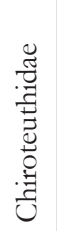 & 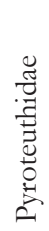 & 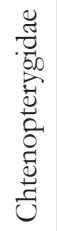 & 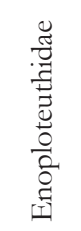 & 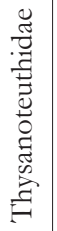 & 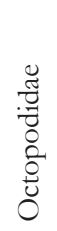 & 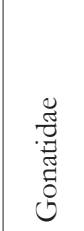 & 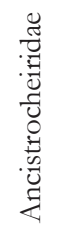 & 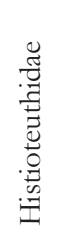 & 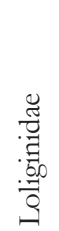 & 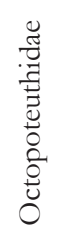 & 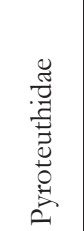 & 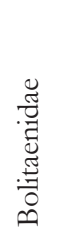 & 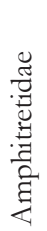 \\
\hline
\end{tabular}


y calamares (Estupiñán et al. 2017), en sus inmediaciones. En todo caso, las abundancias no mostraron diferencias significativas entre las sub-áreas (U M-W: 494; $\mathrm{p}=0,72786$ ), pero sí entre periodos (KW: $H=19,98 ; p=0,0005)$.

Durante los episodios registrados de El Niño en el OPC, entre 1993 y 2007, de septiembre a noviembre, se multiplicaron los remolinos y la Corriente Sur Ecuatorial (océano-costa) incrementó su velocidad, mientras en la Corriente de Colombia (norte-sur) disminuyó, sin variar en el giro anticiclónico, reduciéndose la tasa de dispersión larvaria y la conectividad (Corredor et al. 2011). Esto no fue claro en sep/02, bajo la influencia de El Niño-moderado (jun/02-may/03), detectado en la franja suroccidental hasta la costa del OPC (Devis, 2003); no obstante, un posible indicio del impacto del evento fue la mera presencia de Ommastrephidae, quizás en detrimento de las demás familias, pues pese a la plasticidad y ágil adaptación a condiciones ambientales cambiantes, estos organismos son muy sensibles a las influencias ambientales, con un fuerte efecto en el éxito de desove y eclosión y en el subsiguiente crecimiento y supervivencia paralarval (Rodhouse et al. 2014). En este sentido, Vecchione (1999) anota que el aumento en la abundancia paralarval está relacionado con el incremento térmico, por ejemplo, en el Golfo de California, donde también influyen los volúmenes de zooplancton y la concentración de clorofila (De Silva et al. 2015).

No se explicó la situación análoga en sep/03 (post-El Niño), pero ya que los aspectos reproductivos y ecológicos de la mayoría de cefalópodos son prácticamente desconocidos (Rodhouse et al. 2014; Ruvalcaba et al. 2018), se podría presumir una recuperación lenta de las poblaciones de las familias distintas de Ommastrephidae, tras la ocurrencia de El Niño, hasta llegar a condiciones generales más favorables en 2004, como apuntan los resultados para ese periodo.

El impacto positivo o negativo de El Niño, con cambios considerables en la abundancia, la distribución y los ensamblajes taxonómicos de las paralarvas, debido a las surgencias, remolinos, convergencias y frentes, relacionados con alta productividad biológica a lo largo del Pacífico Oriental, se ha comprobado desde hace tiempo (Vecchione, 1999; Vega et al. 1999; Vega et al. 2000; Granados et al. 2010; Carrasco et al. 2012; De Silva et al. 2015; García et al. 2018; Ruvalcaba et al. 2018).

Se pudo medir la LM de 135 paralarvas (63,4\% del total), predominando las recien eclosionados $(\leq 2 \mathrm{~mm}$ ) (Sweeney et al. 1992) (Figura 2c), con mayor representación en tres periodos hacia la zona nerítica, donde pululan los remolinos (CCCP, 2002; Corredor et al. 2011). Algo análogo ocurre en otras altitudes, incluso, en areas de surgencia que, como los remolinos, tienen una alta productividad primaria y secundaria (Alejo et al. 2013; Aceves et al. 2017; García et al. 2018; Ruvalcaba et al. 2018). En los otros dos periodos, tales tallas fueron más representativas en aguas intermedias y oceánicas, respectivamente, semejante a lo reseñado por Aceves et al. (2017). En el OPC existen surgencias costeras y oceánicas permanentes, sobre todo de agosto a septiembre, sugiriendo su posible efecto sobre la abundancia y la distribución paralarval, al igual que los remolinos.
La LM indica que, en el OPC, la eclosión de las paralarvas ocurre más temprano que en otras áreas (Figura 2, Tabla 1), posiblemente, debido a las mayores temperaturas.

Variación de la abundancia día vs. noche. Hace casi tres décadas (Sweeney et al. 1992), se ha comprobado la migración batimétrica de las paralarvas, abundando más en la noche, en los primeros $200 \mathrm{~m}$, particularmente, en los estratos superficiales, sobre o en la termoclina, en ocasiones, asociadas a los procesos de surgencia, según varios estudios en el Pacífico oriental (Vega et al. 2000; Granados et al. 2010; Ibañez et al. 2012; Ruvalcaba et al. 2018). En el OPC, la abundancia general nocturna superó la diurna ( 83,2 vs. 16,8\%); si bien no se efectuaron muestreos estratificados es plausible aludir a la migración nictimeral, como también lo hicieron Rosales \& López (2007), en sep/05, aunque no se descarta que la noche, probablemente, restrinja la capacidad de las larvas para evitar las redes.

En todo caso, considerando la relación entre estaciones día:noche (60:73) y las abundancias, no hubo diferencias significativas entre las sub-áreas (U M-W: 1348; $\mathrm{p}=0,1031)$, ni entre los periodos (K-W: $\mathrm{H}=2,66 ; \mathrm{p}=0,10318)$.

Análisis estadístico. El análisis factorial explicó satisfactoriamente la varianza acumulada en la relación abundancia-variables abióticas en los dos primeros componentes (61,0-83,0\%). Las correlaciones más altas para la salinidad y la temperatura fueron $-0,86$ a 0,93 y $-0,69$ a 0,86 , respectivamente. Para la relación día:noche variaron entre $-0,63$ y 0,85 (en general 0,58 ) (Tabla 2), indicando efectos positivos y negativos de estas variables en la abundancia paralarval, con variaciones/periodo, seguramente dependiendo de las condiciones particulares en cada caso.

El análisis de agrupamiento abundancia-variables abióticas mostró inter-anualmente dos conjuntos de estaciones (día vs. noche), interiormente, asociados por sub-áreas o estaciones mezcladas (Figura 3), corroborando la relevancia nictimeral y las características termo-halinas en la distribución espacio-temporal de las paralarvas, en el contexto complejo de la variabilidad ambiental en el OPC; no obstante, su distribución en todo el OPC no concuerda con lo expresado por Boyle \& Rodhouse (2005) y Jereb \& Roper (2005), quienes aseveran que la mayoría de especies de cefalópodos son estenohalinas y euritérmicas, por lo que la salinidad sería uno de los principales determinantes abióticos de su distribución geográfica, como también lo refieren Pardo et al. (2016), para los fiordos chilenos, conocidos ecosistemas de alta productividad, con gran fluctuación y variabilidad espacial de la salinidad.

En este contexto, puesto que la comunidad paralarval estuvo dominada por Ommastrephidae, quizás sobre ésta influyó más la temperatura, pues es un factor primordial para su sobrevivencia y crecimiento; su desarrollo óptimo está entre 15,0 y $29,0^{\circ} \mathrm{C}$ y, en aguas costeras de Baja California, las mayores capturas de paralarvas de esta familia ocurren entre 20,5 y $24,0^{\circ} \mathrm{C}$ (Vecchione, 1999), dentro del rango en el OPC $\left(24,4-29,7^{\circ} \mathrm{C}\right)$, durante este estudio. 
Tabla 2. Correlaciones significativas en los componentes 1 y 2 del análisis de factores por componentes principales, para el estudio de las paralarvas en el OPC.

\begin{tabular}{|c|c|c|c|c|c|c|c|}
\hline \multirow[b]{2}{*}{ Periodo } & \multicolumn{3}{|c|}{ Variabilidad explicada (\%) } & \multicolumn{2}{|c|}{ Componente 1} & \multicolumn{2}{|c|}{ Componente 2} \\
\hline & Componente 1 & Componente 2 & Total & Variable & Correlación & Variable & $\begin{array}{l}\text { Cor- } \\
\text { relación }\end{array}$ \\
\hline \multirow{3}{*}{ 23.Jun-12.Jul.01 } & \multirow{3}{*}{40,0} & \multirow{3}{*}{37,0} & \multirow{3}{*}{77,0} & \multirow{3}{*}{ Día:noche } & \multirow{3}{*}{$-0,82$} & Salinidad & 0,90 \\
\hline & & & & & & Temperatura & $-0,69$ \\
\hline & & & & & & Abundancia & 0,66 \\
\hline \multirow{2}{*}{ 27.Ago-15.Sep.01 } & \multirow{2}{*}{42,0} & \multirow{2}{*}{41,0} & \multirow{2}{*}{83,0} & Salinidad & $-0,86$ & Abundancia & 0,97 \\
\hline & & & & Día:noche & $-0,63$ & Temperatura & 0,85 \\
\hline \multirow{2}{*}{ 03-22.Sep.02 } & \multirow{2}{*}{43,0} & \multirow{2}{*}{27,5} & \multirow{2}{*}{70,5} & Temperatura & 0,86 & Abundancia & $-0,67$ \\
\hline & & & & Salinidad & $-0,82$ & Día:noche & 0,85 \\
\hline \multirow{3}{*}{ 01-21.Sep.03 } & \multirow{3}{*}{39,0} & \multirow{3}{*}{30,5} & \multirow{3}{*}{69,5} & Salinidad & $-0,90$ & \multirow{3}{*}{ Abundancia } & \multirow{3}{*}{0,80} \\
\hline & & & & Temperatura & 0,77 & & \\
\hline & & & & Día:noche & 0,72 & & \\
\hline \multirow{3}{*}{ 18.Sep-08.Oct.04 } & \multirow{3}{*}{34,0} & \multirow{3}{*}{27,0} & \multirow{3}{*}{61,0} & \multirow{3}{*}{ Salinidad } & \multirow{3}{*}{0,93} & Abundancia & 0,73 \\
\hline & & & & & & Temperatura & $-0,66$ \\
\hline & & & & & & Día:noche & 0,52 \\
\hline \multirow{3}{*}{ General } & \multirow{3}{*}{32,0} & \multirow{3}{*}{26,0} & \multirow{3}{*}{58,0} & Abundancia & 0,81 & \multirow{3}{*}{ Salinidad } & \multirow{3}{*}{0,78} \\
\hline & & & & Temperatura & $-0,73$ & & \\
\hline & & & & Día:noche & 0,58 & & \\
\hline
\end{tabular}
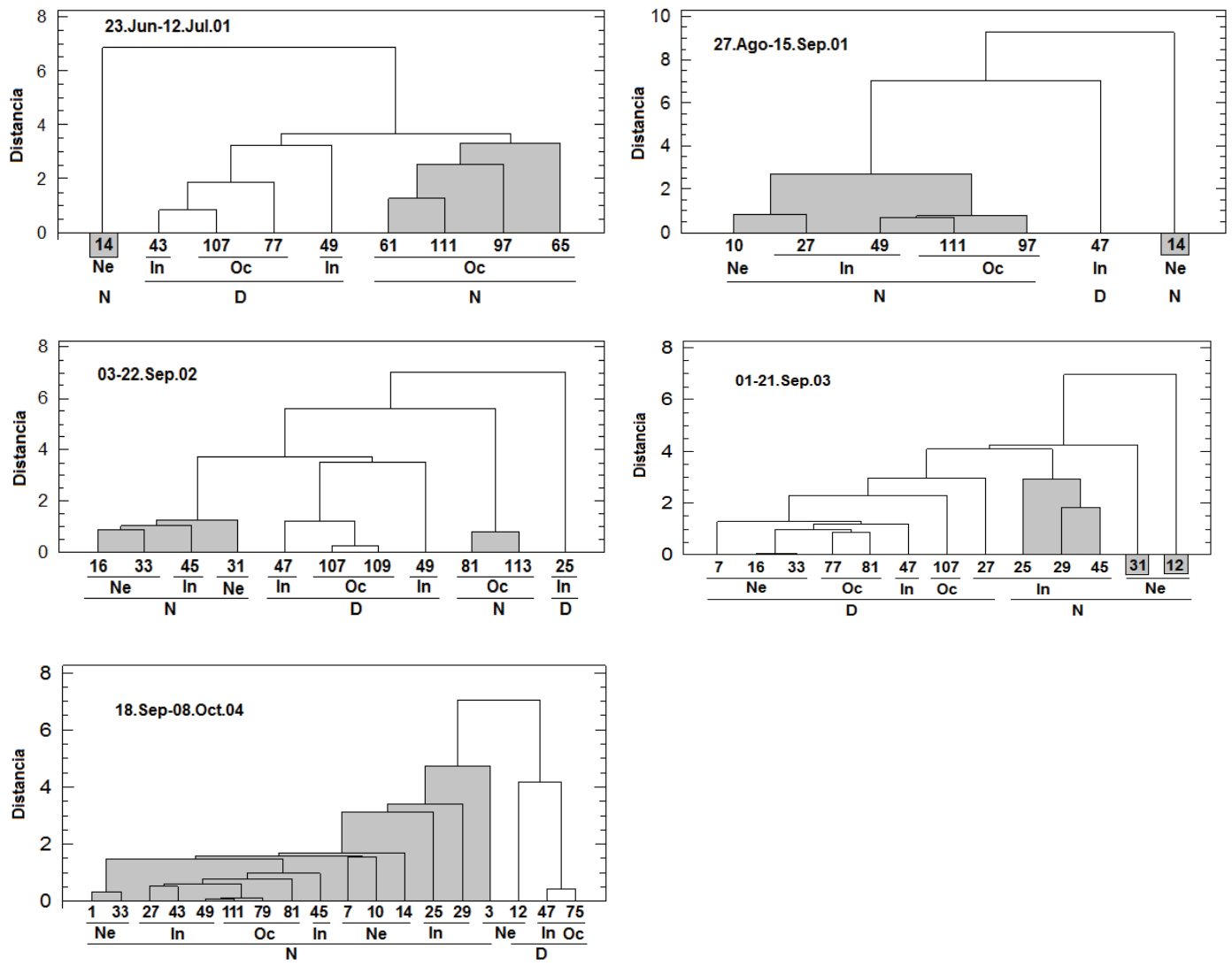

Figura 3. Dendrograma modo-Q mostrando el agrupamiento entre las estaciones de muestreo, con base en las abundancias paralarvales y las variables abióticas en los cinco periodos evaluados en el OPC. Sub-áreas: Ne= nerítica, In: intermedia, Oc: oceánica. D: día, N: noche. 
Para comprender en profundidad la dinámica de la comunidad paralarval en el OPC, en los próximos estudios, se sugiere profundizar en su taxonomía, mediante técnicas moleculares e incluir en los análisis la productividad primaria y secundaria y las relaciones tróficas de las paralarvas con otros zoopláncteres, considerando muestreos estratificados. Además, contemplar una cronología más robusta, que incluya un registro de las corrientes simultáneo con los muestreos en diferentes meses, para poder conocer la estructura comunitaria y establecer el patrón y la magnitud de la dispersión larvaria, impulsada por las corrientes y la conectividad poblacional entre los individuos de cada localidad (Rodhouse $e t$ al. 2014; Villanueva et al. 2016). También falta establecer la aparición de otras familias, diferentes de Ommastrephidae, lo que aportará al conocimiento de los ciclos biológicos, para comprender la dinámica poblacional y, especialmente estimar el reclutamiento de potenciales taxa comerciales, como sugieren Rodhouse et al. (2014).

De la presente investigación, se puede concluir que, muy probablemente, en el OPC, la distribución horizontal de las paralarvas se deba a su alto potencial de dispersión meso-escalar, mediado por la acción combinada del giro geostrófico, la Corriente Sur Ecuatorial y la Corriente de Colombia. En este aspecto, también es relevante el gradiente termo-halino, pues aparentemente los desoves y eclosión tienden a ser más recurrentes hacia la zona nerítica de mayor temperatura. En la distribución, además influye la variación circadiana, con mayores abundancias nocturnas, posiblemente relacionadas con las migraciones verticales de las paralarvas.

La eclosión de las paralarvas en el OPC acontece más temprano que en otras áreas del Pacífico Americano, atribuíble a las mayores temperaturas.

Además de otras variables no consideradas aquí, e.g., la disponibilidad de alimento y la presencia de depredadores, la variación inter-anual en la estructura termohalina y la composición taxonómica debe responder a los cambios oceanográficos y climáticos en el OPC, resaltándose la influencia de El Niño-moderado que, aparentemente, en detrimento de las demás familias, benefició a Ommastrephidae, que por su abundancia y distribución parece ser la base de la comunidad de cefalópodos en esta área.

Agradecimientos. A la Dirección General Marítima (DIMAR) y Centro Control de Contaminación del Pacífico (CCCP), por el suministro del listado básico de los datos abióticos y las muestras biológicas. A la Vicerrectoría de Investigaciones de la Universidad Militar Nueva Granada, por la financiación del proyecto CIAS 134: Dinámica ecológica del zooplancton en el Océano Pacífico Colombiano. Cruceros 2007-2010 de la serie ERFEN. Conflicto de intereses: El manuscrito fue preparado por los autores, quienes declaran que no existe ningún conflicto de intereses que ponga en riesgo la validez de los resultados presentados.

\section{REFERENCIAS}

1. ACEVES, G.; DE SILVA, R.; CRUZ, I.; DURAZO, R.; AVENDAÑO, R. 2017. Influence of the oceanographic dynamic in size distribution of cephalopod paralarvae in the southern Mexican Pacific Ocean (rainy seasons 2007 and 2008). Lat. Am. J. Aquat. Res. (Chile). 45(2):356-369. https://doi.org/10.3856/vol45-issue2-fulltext-11

2. ALEJO, M.C.; GARCÍA, R.; HERRERA, J. 2013. Paralarvas y juveniles de cefalópodos en el Pacífico sur de México. Hidrobiológica (México). 23(2):250-264.

3. ALEJO-PLATA, C.; GARCÍA-GUILLÉN, R.; HERRERAGALINDO, J. 2012. Paralarvas y juveniles de Octopus bimaculatus (Cephalopoda: Octopodidae) en el Pacífico sur de México. Rev. Biol. Mar. Ocean. 47(2):359-365.https:// doi.org/10.4067/S0718-19572012000200019

4. ALLCOCK, L.; BARRATT, I. 2014. Ancistrocheirus lesueuri. The IUCN Red List of Threatened Species 2014 (Inglaterra): e.T163050A966613. http://dx.doi.org/10.2305/IUCN. UK.2014-.RLTS.T163050A966613.en

5. ARKHIPKIN, A.I. 1997. Age and growth of the mesopelagic squid Ancistrocbirus lesueurii from the centraleast Atlantic based on statolith microstructure. Mar. Biol. (Estados Unidos). 129(1):103-111.

6. AYÓN, P. 2018. Variabilidad espacial y temporal de paralarvas de cefalópodos (1980-2014) en el mar peruano. Bol. Inst. Mar. Perú. (Perú). 33(2):253-265.

7. BOWER, J.R; TAKAGI, S. 2004. Summer vertical distribution of paralarval gonatid squids in the northeast Pacific. J. Plankton Res. (Inglaterra). 26(8):851-857.https://doi. org/10.1093/plankt/fbh081

8. BOYLE, P.; RODHOUSE, P. 2005. Cephalopods. Ecology and Fisheries. Blackwell Publishing. (Estados Unidos). 464p.

9. CARDOSO, F; BALTAZAR, P.; BAUTISTA, J. 2005. The early development of the Patagonian squid Loligo gabi D'Orbigny, 1835 in Peruvian Waters (Cephalopoda: Loliginidae). Rev. Peru. Biol. (Perú). 12(3):369-376.

10. CARRASCO, S.; MALTRAIN, R.; VILLENAS, F.; VEGA, M. 2012. New records of early life-stages of cephalopods in the Chiloé Interior Sea. Lat. Am. J. Aquat. Res. (Chile). 40(1):229-235. https://doi.org/10.3856/vol40-issue1fulltext-22

11. CENTRO CONTROL CONTAMINACIÓN DEL PACÍFICO, CCCP. 2002. Compilación oceanográfica de la Cuenca 
Pacífica Colombiana. Centro Control Contaminación del Pacífico. Ed. Imágenes de la Naturaleza, Cali (Colombia), 109p.

12. CORREDOR, A.; ACOSTA, A.; GASPAR, P.; CALMET'TES, B. 2011. Variation in the surface currents in the Panama bight during El Niño and La Niña events from 1993 to 2007. Bol. Invest. Mar. Cost. (Colombia). 40(Supl. Esp.):3356. https://doi.org/10.25268/bimc.invemar.2011.40.0.127

13. DE LA HOZ, J.; DUARTE, L.O.; MANJARRÉS, L. 2017. Estadísticas de desembarco y esfuerzo de las pesquerías artesanales e industriales de Colombia entre marzo y diciembre de 2017. Autoridad Nacional de Acuicultura y Pesca (AUNAP), Santa Marta (Colombia). 84p.

14. DE SILVA, R.; FRANCO, C.; HOCHBERG, F.G.; GODÍNEZ, E.; AVENDAÑO, R.; GÓMEZ, J.; ROBINSON, C.J. 2015. Cephalopod paralarval assemblages in the Gulf of California during 2004-2007. Mar. Ecol. Prog. Ser. (Alemania). 520:123-141. https://doi.org/10.3354/ meps11074

15. DEVIS, A. 2003. Evolución del evento El Niño 2002-2003 y efectos sobre la cuenca del Pacífico colombiano y la bahía de Tumaco. Bol. Cien. CCCP (Colombia). 10:12-32.

16. DOMÍNGUEZ, J.F.; CEBALLOS, B.P.; HOCHBERG; F.G.; ARELLANO, M. 2013. A new record in a wellestablished population of Octopus bubbsorum (Cephalopoda: Octopodidae) expands its known geographic distribution range and maximum size. Amer. Malacol. Bull. (Estados Unidos). 31(1):95-99. http://dx.doi. org/10.4003/006.031.0122

17. ESTUPIÑÁN, C.; GALVÁN, F.; TAMBURÍN, E.; SÁNCHEZ, A.; VILLALOBOS, D.J.; MURILLO, N.; BESSUDO, S.; ESTUPIÑÁN, J.F. 2017. Trophic inference in two sympatric sharks, Sphyrna lewini and Carcharbinus falciformis (Elasmobranchii: Carcharhiniformes), based on stable isotope analysis at Malpelo Island, Colombia. Acta Ichtyol. Pisc. (Polonia). 47(4):357-364. http://dx.doi.org/10.3750/ AIEP/02177

18. GARCÍA, R.M.; DE SILVA, R.; AVENDAÑO, R. 2018. Seasonal changes in paralarval cephalopod communities on the southwest coast of Baja California Sur (spring and autumn 2003). Cienc. Mar. (México). 44(2):107-123. http:// dx.doi.org/10.7773/cm.v44i2.2761

19. GRANADOS, J.; SILVA, R.; CAMARILLO, S.; HOCHBERG, F.; DURAZO, R.; AVENDAÑO, R.; HERNÁNDEZ, M. 2010. Composición de especies y patrones de distribución de paralarvas de calamar 1996-1999. En: Gaxiola, G.; Durazo, R. (eds.). Dinámica del ecosistema pelágico frente a Baja California, 1997-2007. Diez años de investigaciones mexicanas de la Corriente de California. Sec. Med. Amb. Rec. Nat. México D. F. (México). p.453-467.

20. HENDRICKX, M.E.; URBANO, B.; ZAMORANO, P. 2015. Distribution of pelagic squids Abraliopsis Joubin, 1896 (Enoploteuthidae) and Pterygioteuthis P. Fischer, 1896 (Pyroteuthidae) (Cephalopoda, Decapodiformes, Oegopsida) in the Mexican Pacific. ZooKeys (Bulgaria). 537:51-64. http://dx.doi.org/10.3897/zookeys.537.6023

21. IBAÑEZ, C.M.; ARGUELLES, J.; YAMASHIRO, C.; ADASME, L.; CÉSPEDES, R.; POULIN, E. 2012. Spatial genetic structure and demographic inference of the Patagonian squid Doryteuthis gahi in the south-eastern Pacific Ocean. J. Mar. Biol. Assoc. UK. (Inglaterra), 92(1):197-203. https:// doi.org/10.1017/S0025315411000440

22. JEREB, P.; ROPER, C.F.E. 2005. Cephalopods of the world. An annotated and illustrated catalogue of species known to date. Chambered nautiluses and sepioids (Nautilidae, Sepiidae, Sepiolidae, Sepiadariidae, Idiosepiidae and Spirulidae), vol. 1. FAO Species Catalogue for Fishery Purposes, (Italia). 262p.

23. JORGENSEN, E.M. 2007. Identification, distribution and relative abundance of paralarval gonatid squids (Cephalopoda: Oegopsida: Gonatidae) from the Gulf of Alaska, 2001-2003. J. Moll. Stud. (Inglaterra). 73(2):155165.

24. PARDO, M.C.; IBÁNEZ, C.M.; RUIZ, J.F.; BUSTOS, C.A.; PENA, F.A.; LANDAETA, M.F. 2016. Paralarvae of cephalopods in channels and fjords of the southern tip of Chile (46-53॰S). Fish. Res. (Holanda). 173:175-182. http:// dx.doi.org/10.1016/j.fishres.2015.07.001

25. RODHOUSE, P.G.K.; PIERCE, G.J.; NICHOLS, O.C.; SAUER, W.H.H.; ARKHIPKIN, A.I.; LAPTIKHOVSKY, V.V.; LIPIHNSKI, M.R.; RAMOS, J.E.; GRAS, M.; KIDOKORO, H.; SADAYASU, K.; PEREIRA, J.; LEFKADITOU, E.; PITA, C.; GASALLA, M.; HAIMOVICI, M.; SAKAI, M.; DOWNEY, N. 2014. Environmental effects on cephalopod population dynamics: Implications for management of fisheries. Adv. Mar. Biol. (Estados Unidos). 67:99-234. https://doi.org/10.1016/B978-0-12-800287-2.00002-0

26. ROPER, C.F.E.; JEREB, P. 2010. Cephalopods of the world, FAO Species Catalogue for Fisheries Purpouses. Roma (Italia). 4(2):1-183.

27. ROSALES, N.; LÓPEZ, R. 2007. Paralarvas de calamares y pulpos capturadas en el océano pacífico colombiano. Estudio preliminar: periodo 02-25.Sep.05. Rev. Fac. Cien. (Colombia). 3(1):145-161. 
28. RUVALCABA, E.D.; SÁNCHEZ, L.; BEIERB, E.; GODÍNEZ, V.M.; BARTOND, E.D.; PACHECO, M.R. 2018. Effects of mesoscale structures on the distribution of cephalopod paralarvae in the Gulf of California and adjacent Pacific. Deep-Sea Res. Part I. (Inglaterra). 131: 62-74. https://doi. org/10.1016/j.dsr.2017.11.005

29. SMITH, P.E.; RICHARDSON, S.L. 1979. Técnicas modelo para prospección de huevos y larvas de peces pelágicos. Doc. Téc. Pes. FAO, Roma (Italia). 175p.

30. SWEENEY, M.J.; ROPER, C.F.E.; MANGOLD, K.M.; BOLETZKY, S.V. (eds.) 1992. "Larval" and juvenile cephalopods: A manual for their identification. Smithson. Contrib. Zool. (Estados Unidos). 513:282p.

31. VECCHIONE, M. 1999. Extraordinary abundance of squid paralarvae in the tropical eastern Pacific Ocean during El Niño of 1987. Fish. Bull. (Estados Unidos). 97:1025-1030.

32. VEGA, M.; ROCHA, F.; GUERRA, A.; OSORIO, C.; MARÍN, V. 1999. Estudio preliminar de las paralarvas de cefalópodos frente a la península de Mejillones. Ami. Moll. (Chile). 7:2530.

33. VEGA, M.; ROCHA, F.; OSORIO, C. 2000. Variaciones espaciales y temporales de paralarvas de cefalópodos en el Canal Moraleda (43 S), sur de Chile. Rev. Cienc. Tecnol. Mar. (Chile). 23:69-82.

34. VILLANUEVA, R.; VIDAL, E.A.G.; FERNÁNDEZÁLVAREZ, F.A.; NABHITABHATA, J. 2016. Early mode of life and hatchling size in cephalopod molluscs: Influence on the Species Distributional Ranges. PLoS ONE 11(11):e0165334. https://doi.org/10.1371/journal. pone. 0165334

35. VOSS, N.A.; STEPHEN, S.J.; DONG, Z. 1992. Family Cranchiidae Prosch, 1849. Smithson. Contr. Zool. (Estados Unidos). 513:187-210.

36. YOUNG, R.E.; MANGOLD, K.M.; VECCHIONE, M. 1992. The Enoploteuthid group of families. En: Sweeney, M.J.; Roper, C.F.E.; Mangold, K.M.; Clarke, M.R.; Boletzky, S.V. (eds.) Larval and juvenile cephalopods. a manual for their identification. Smithson. Contr. Zool. (Estados Unidos). 12:91-112. 